\title{
Valoración de las necesidades de atención psicológica en pacientes en hemodiálisis mediante indicadores de ansiedad y depresión
}

\author{
Rosa María Salas Muñoz, Antonio José Fernández Jiménez \\ Centro de Diálisis Sierra Este. Sevilla. España
}

Como citar este artículo: Salas-Muñoz RS, Fernández-Jiménez AJ. Valoración de las necesidades de atención psicológica en pacientes en hemodiálisis mediante indicadores de ansiedad y depresión. Enferm Nefrol. 2019 Abr-Jun;22(2): 177-84

\section{Resumen}

Introducción: Numerosos trabajos sugieren relaciones entre la enfermedad renal crónica severa y trastornos del estado de ánimo.

Objetivo: Valorar las necesidades de atención psicológica de la población en hemodiálisis a partir de indicadores objetivos de ansiedad y depresión.

Material y Método: Estudio descriptivo transversal entre octubre de 2016 y marzo de 2018, con una muestra de 103 pacientes a los que se entrevistó y se les aplicó el inventario de depresión de Beck y el inventario de Ansiedad Estado-Rasgo. Se registró el sexo, edad y el tiempo que cada persona llevaba en diálisis.

Resultados: Un 8,7\% de la muestra presentó niveles de depresión grave, frente a un $60,2 \%$ con depresión mínima. El 23,3\% obtuvo niveles leves de depresión y un $7,8 \%$ depresión moderada.

Un 25,24\% de la muestra presentaban ansiedad elevada, un $37,86 \%$ ansiedad moderada y un $36,89 \%$ baja ansiedad.

Según cuestionarios realizados, 92 de los 103 participantes, describieron la existencia de un evento traumático en sus vidas, junto con estrategias de afrontamiento pasivo/evitativas.

\section{Correspondencia:}

Rosa Ma Salas Muñoz

Centro de Diálisis Sierra Este

Avenida de la Innovación. 2. 41020 Sevilla

E-mail: psicologia@dialisisandaluza.es
Conclusiones: A partir de los resultados observados en los que es patente la presencia de ansiedad y depresión en los pacientes en hemodiálisis, podemos apreciar la importancia de disponer para ellos de un tratamiento psicológico.

PALABRAS CLAVE: hemodiálisis; ansiedad; depresión; alexitimia; psicología; trastornos emocionales.

\section{Assessment of needs of psychological care in hemodialysis patients using indicators of anxiety and depression}

Introduction: Several studies suggest relationships between severe chronic kidney disease and mood disorders.

Objective: To assess the psychological care needs of the hemodialysis population based on objective indicators of anxiety and depression.

Material and Method: A cross-sectional descriptive study between October 2016 and March 2018 was carried out, with a sample of 103 patients who were interviewed and applied the Beck depression inventory and the State-Trait Anxiety Inventory. Gender, age and time on dialysis were recorded.

Results: $8.7 \%$ of the sample showed levels of major depression, compared with $60.2 \%$ with minimal depression. $23.3 \%$ obtained mild levels of depression and $7.8 \%$ moderate depression. $25.24 \%$ of the sample had high anxiety, $37.86 \%$ moderate anxiety and $36.89 \%$ low anxiety. 
According to questionnaires, 92 of the 103 participants described the existence of a traumatic event in their lives, along with passive/avoidant coping strategies.

Conclusions: Based on the results observed in which the presence of anxiety and depression is evident in patients on hemodialysis, it is required to have an adequate psychological treatment.

KEYWORDS: hemodialysis; anxiety; depression; alexithymia; psychology; emotional disorders.

\section{Introducción}

El tratamiento de la insuficiencia renal crónica mediante hemodiálisis supone para el paciente asumir una situación psicológica compleja, ya que coexisten fuertes condicionantes contradictorios entre sí, asociados por un lado a la dependencia, y al autocontrol, por otro. La dependencia viene marcada por el propio carácter del tratamiento, tan necesario para que estas personas se mantengan con vida, así como por el tiempo que requiere, sumado al necesario para el transporte, y a las limitaciones de movilidad geográfica. El autocontrol tiene que ver con las restricciones hídricas y alimenticias, así como por el complejo tratamiento farmacológico requerido. Todas estas circunstancias, se ven además agravadas por la comorbilidad y enfermedades asociadas ${ }^{1,2}$.

En definitiva, todas las circunstancias descritas implican grandes pérdidas de autonomía y, fundamentalmente, la pérdida de la condición de personas "sanas" para siempre. Todo lo que tiene que ver con pérdidas en la vida de las personas, supone un proceso de duelo que suele asociarse a síntomas de índole depresiva ${ }^{3,4}$.

Por otra parte, el tratamiento en hemodiálisis supone una cronicidad a veces difícil de asumir por las personas, al no poder visualizar el final del mismo y verse libres de sus requerimientos. La esperanza de un trasplante renal puede aliviar los sentimientos de angustia que esta situación despierta ${ }^{5}$ pero, por desgracia, dicha posibilidad queda descartada para muchas personas 0 se retrasa en el tiempo más de lo deseable.

Todos los factores mencionados, nos hacen sospechar que estamos ante personas con una alta probabilidad de padecer síntomas depresivos y ansiedad, asociados tanto a las mencionadas pérdidas que deben asumir, como a la incertidumbre ante un delicado estado de salud que hace que su futuro sea dependiente de un tratamiento médico complejo.

Otro aspecto relevante para este estudio es la consideración de la persona como un todo indisociable donde factores físicos, mentales y emocionales fluctúan interrelacionándose a lo largo de todo el ciclo vital. De forma que, personas que han tenido vidas cargadas de sufrimiento, van a tener también, con una alta probabilidad, cuerpos donde este sufrimiento se ve reflejado en forma de enfermedades de diversa índole ${ }^{6}$. Por ello, cabe suponer que las personas en hemodiálisis van a padecer un alto grado de deterioro psicológico, no sólo por los condicionantes antes descritos, sino también por las situaciones vitales y relacionales que experimentan, o que han vivido en el pasado, y que contribuyen al sufrimiento emocional y físico en sus vidas.

Nos planteamos como objetivo conocer hasta qué punto estas personas presentan alteraciones emocionales, que pudieran requerir de un abordaje psicológico como tratamiento complementario para mejorar su calidad de vida y su estado general de salud.

\section{Material y Método}

Estudio observacional descriptivo transversal realizado entre los meses de octubre de 2016 y marzo de 2018, en el centro de hemodiálisis, Diálisis Andaluza, Sevilla.

\section{Participantes}

Contamos con una muestra de 103 pacientes, hombres y mujeres mayores de edad, que cumplieron todos como criterio de inclusión el estar en condiciones físicas y psicológicas adecuadas para poder responder a las preguntas de los instrumentos utilizados.

En cuanto a la edad de los sujetos, se realizaron tres agrupaciones, para poder comprobar si esta variable tiene alguna influencia en el estado psicológico: 1) menores de 45 años. 2) entre 45 y 65 años. 3) mayores de 65 años.

Se recogió también el tiempo que cada persona llevaba en diálisis, dividiéndose la muestra en tres subgrupos: 1) menos de 6 meses en diálisis, 2) entre 6 meses y 4 años en diálisis, 3) más de 4 años en diálisis.

\section{Instrumentos}

A todos los sujetos de la muestra se le aplicaron los siguientes cuestionarios: 
1) Cuestionario de Ansiedad Estado-Rasgo (STAI) ${ }^{7}$. Aunque este cuestionario consta de dos escalas independientes: Ansiedad-Estado y Ansiedad-Rasgo, la escala aplicada fue la segunda. El motivo es que la escala Ansiedad-Estado evalúa un estado emocional transitorio, caracterizado por sentimientos subjetivos de atención y aprensión circunscritos al momento de la evaluación y que se acompañan de la hiperactividad del sistema nervioso autónomo. En diálisis, es habitual encontrar momentos de mucha tensión y aprensión relacionados con la conexión a la máquina y, en general, las personas son especialmente sensibles ante cualquier incidente propio o ajeno, por leve que sea, por lo que consideramos que esta puntuación podría estar sesgada por el contexto en el que se realiza la evaluación, siendo poco representativa de la tendencia de la persona a manifestar la ansiedad como respuesta habitual en su vida cotidiana. Por el contrario, la puntuación de Ansiedad-Rasgo señala una propensión ansiosa, relativamente estable, que caracteriza a los individuos con tendencia a percibir las situaciones como amenazadoras. Además, en anteriores estudios se han encontrado correlaciones positivas entre las puntuaciones de ambas escalas ${ }^{8}$. Los puntajes de este cuestionario para cada sujeto quedan transformadas en un único valor decatípico que va de 1 a 10. Para mayor facilidad interpretativa, hemos considerado la agrupación de las puntuaciones de la muestra atendiendo también a tres tramos de ansiedad: 1) 1-4, baja. 2) 5-7 moderada. 3) 8-10 elevada.

2) Cuestionario de Depresión de Beck para pacientes médicos (BDI-FS) ${ }^{9}$. Consta de 7 ítems, extraídos del BDI-II, para evaluar depresión en adolescentes y adultos con un diagnóstico médico. Permite conocer los síntomas cognitivos y afectivos vinculados a la depresión, excluyendo aquellos que podrían deberse a trastornos o enfermedades médicas. Esta prueba se diseñó para reducir el número de falsos positivos de depresión en personas cuyos síntomas somáticos o conductuales, atribuibles a trastornos orgánicos o relacionados con el consumo de sustancias, podrían confundir el diagnóstico. Las puntuaciones arrojadas por este inventario se dividieron en cuatro tramos de depresión establecidos por los propios autores del mismo: 1) 0-3, mínima. 2) 4-8, leve. 3) 9-12, moderada. 4) 13-21, grave.

\section{3) Cuestionario de elaboración propia} sobre experiencias vitales. Ver ANEXO 1. Consistió en una serie de preguntas sobre diferentes etapas del ciclo vital a las que cada persona respondía verbalmente y cuyas respuestas eran anotadas por la evaluadora para su posterior revisión y recuento de eventos significativos en la vida de cada uno de ellos.

\section{Procedimiento}

Tras obtener la aprobación del comité de ética y el consentimiento informado de los pacientes, los tres cuestionarios mencionados se aplicaron en el contexto de una entrevista individual. Todas las entrevistas fueron realizadas por la psicóloga del centro de diálisis. Según la disponibilidad de cada paciente, tuvieron lugar bien antes de que diera comienzo la sesión de diálisis o durante la misma.

\section{Análisis estadístico}

Se realizó un análisis descriptivo de frecuencias para las variables estudiadas. Para las comparaciones de medias se recurrió a la prueba $T$ de Student para muestras independientes en el caso de variables con dos grupos (sexo), y a un análisis de la varianza (ANOVA) con el correspondiente test Post-hoc (Tukey) en el caso de variables estratificadas en más de dos grupos. Para evaluar el grado de asociación entre ansiedad y depresión se aplicó el coeficiente de correlación de Pearson.

\section{Resultados}

\section{Datos demográficos}

La muestra estuvo compuesta por 42 mujeres y 61 hombres. La distribución en cuanto a la edad y tiempo en diálisis puede observarse en la Tabla 1.

\section{Resultados BDI-FS}

Las comparaciones de medias por grupos de sexo, edad o tiempo en diálisis no mostraron diferencias estadísticamente significativas: $p=0,451, p=0,237$ y $p=0,4$ respectivamente.

Atendiendo al total de la muestra, los porcentajes de las puntuaciones obtenidas pueden observarse en la Figura 1.

\section{Resultados STAI}

Los resultados no mostraron diferencias estadísticamente significativas al comparar las medias para los grupos

Tabla 1. Distribución de frecuencias según edad y tiempo en diálisis.

\begin{tabular}{|ccc|ccc|}
\hline \multicolumn{3}{|c|}{ EDAD } & \multicolumn{3}{|c|}{ TIEMPO EN DIÁLISIS } \\
\hline$<45$ años & $45-65$ años & $>65$ años & $<6$ meses & 6 meses-4 años & $>4$ años \\
\hline 18 sujetos & 41 sujetos & 44 sujetos & 30 sujetos & 57 sujetos & 16 sujetos \\
\hline
\end{tabular}




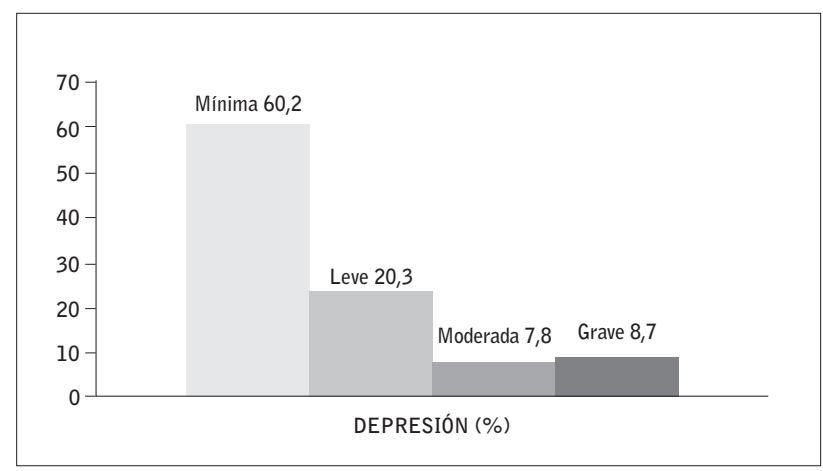

Figura 1. Puntuaciones BDI-FS

de sexo $(p=0,606)$, edad $(p=0,172)$ o tiempo en diálisis $(p=0,298)$.

Los porcentajes de puntuaciones del total de la muestra para la escala de ansiedad-rasgo pueden observarse agrupadas por estratos en la Figura 2, o bien en forma de decatipos en la Figura 3.

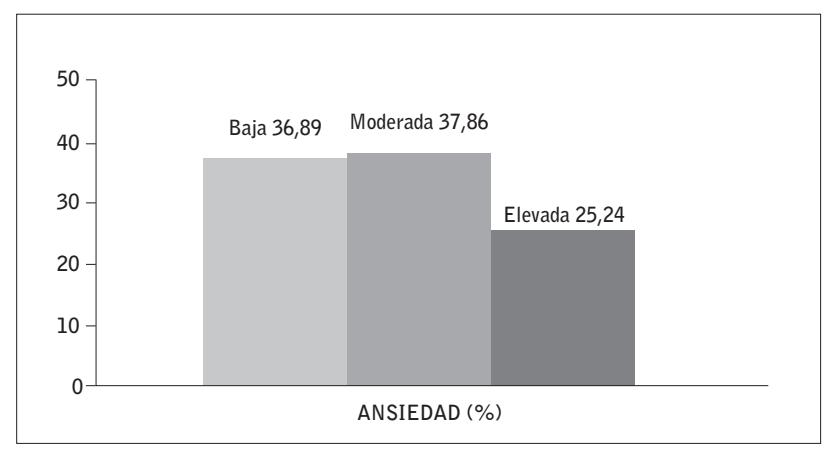

Figura 2. Puntuaciones STAI.

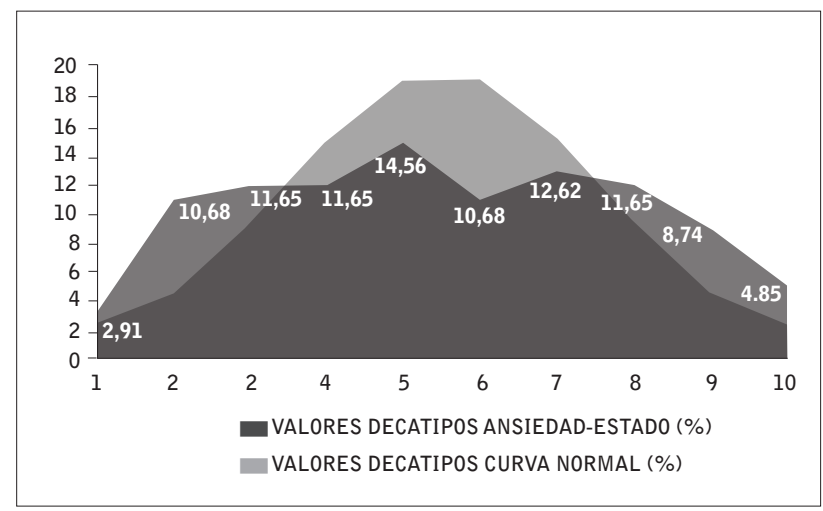

Figura 3. Puntuaciones decatipos STAI.

\section{Correlación BDI-FS y STAI}

La correlación de Pearson entre las puntuaciones de ambas pruebas arrojó un coeficiente positivo con valor $r p=0,699$, que resultó ser estadísticamente significativo $(p<0,01)$. La dispersión de los resultados de depresión y ansiedad puede observarse gráficamente en Figura 4.

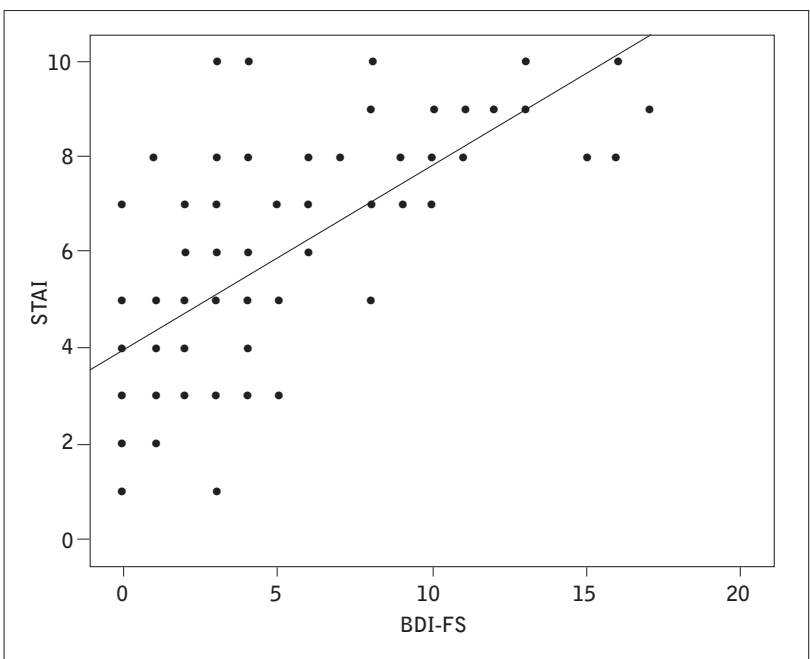

Figura 4. Cuadro de dispersión de las puntuaciones de depresión y ansiedad.

\section{Cuestionario de experiencias vitales}

Del total de los 103 pacientes entrevistados, 92 habían sufrido al menos una situación potencialmente traumática en sus vidas diferente a la de padecer una enfermedad crónica, del tipo que se describe a continuación:

- Maltrato y/o abuso físico y/o psicológico por parte de alguno de sus progenitores y/o de sus parejas (70 personas).

- Fallecimiento por enfermedad o accidente de algún hijo/a (34 personas).

- Situaciones donde han visto peligrar su vida o la de seres queridos cercanos (67 personas).

- Situaciones de negligencia y/o abandono por parte de sus progenitores cuando eran menores de edad sin posibilidad de auxilio por parte de otros adultos (54 personas).

\section{Discusión}

Los resultados de este estudio muestran que un 16,5\% de los pacientes en hemodiálisis muestran sintomatología depresiva clínicamente relevante y un $25 \%$ manifiesta un componente ansioso patológico como parte de sus respuestas cotidianas. Consideramos que dichas 
alteraciones, sin el apoyo de un tratamiento psicológico especializado, podrían estar afectando negativamente a la manera en que estos pacientes afrontan su enfermedad y tratamiento médico, como muestran otros estudios donde se pone en relación la sintomatología depresiva y ansiosa con una gran merma en la calidad de vida de los pacientes y su adherencia al tratamiento $0^{10-12}$.

Al comparar nuestros resultados con los obtenidos en otras unidades de hemodiálisis españolas, observamos que existe una alta coincidencia en los porcentajes de puntajes elevados de ansiedad, que rondan una cuarta parte de la muestra ${ }^{13-15}$. Sin embargo, nuestros porcentajes para depresión moderada/severa son menores que los obtenidos en otros estudios, donde superan el $25 \% 8,16$ o incluso el $50 \%$ de la muestra ${ }^{15}$. Pensamos que el motivo podría radicar en las diferencias en el instrumento de evaluación utilizado, ya que aunque se ha considerado que el BDI es una herramienta válida y útil en este tipo de pacientes, especialmente cuando se eliminan del mismo las escalas referidas a síntomas físicos, quedando la prueba reducida de 21 a 15 ítems $^{13}$, nosotros optamos por su versión abreviada para paciente médicos, que consta únicamente de 7 ítems, no sólo por la evitación de los ítems relacionados con la dimensión física de la depresión, sino también para mayor facilidad de aplicación. Dadas las discrepancias con otros estudios, planteamos la posibilidad de que esta versión carezca de la sensibilidad suficiente para la valoración de la depresión en hemodiálisis, o bien, de que pudiera estar evitando la existencia de falsos positivos, interrogante que podría formar parte del objetivo de futuras investigaciones.

También queda patente que existe una relación positiva entre el padecimiento de alteraciones depresivas y ansiosas, por lo que cabe suponer que es frecuente encontrar trastornos mixtos ansioso-depresivos en hemodiálisis, tal y como avalan otros estudios ${ }^{8,17}$. De forma que personas más deprimidas, también tienden a mostrar estrategias de afrontamiento más cargadas de ansiedad ante diferentes circunstancias en sus vidas.

Las alteraciones emocionales detectadas, no se vieron influenciadas por el sexo, ni por la edad, ni por el tiempo que los pacientes llevaban acudiendo al tratamiento mediante hemodiálisis. Esto nos hace pensar que la tendencia de estas personas a padecerlas pudiera tener que ver no sólo con el hecho de estar enfermos y sometidos a este tratamiento, sino con otros factores estresantes presentes en sus vidas. Además, la puesta en marcha de estrategias de afrontamiento poco funcionales basadas en la actitud pasiva y la evitación podría tener que ver con estos resultados, lo que queda pendiente explorar en futuros estudios, apoyando así a otros autores que ponen este hecho en relación con la baja adhesión al tratamiento de estos pacientes y con índices elevados de depresión y ansiedad ${ }^{18-19}$. De esta forma, apoyamos la hipótesis de que el deterioro físico que muchas personas muestran, podría ser un reflejo somático del sufrimiento padecido a lo largo de sus vidas ${ }^{20}$.

No queremos pasar por alto el hecho de que la mayoría de las personas evaluadas obtuvo niveles mínimos tanto de depresión $(83,5 \%)$ como de ansiedad $(74,75 \%)$ de los cuales, el 60 y el 40 por ciento respectivamente, muestra casi total ausencia de esta sintomatología. Este hecho ocurre en contra de lo que cabría esperar dado el delicado estado de salud de estas personas, que conviven con una enfermedad crónica severa que muchas veces coexiste con otras enfermedades, y también a pesar de que, en su gran mayoría, han tenido vidas desestructuradas, protagonizadas por experiencias altamente estresantes a las que hacen frente con estrategias psicológicas poco adaptativas, en su mayoría relacionadas con la evitación y el desarrollo de conductas de dependencia, según se extrae de la entrevistas realizadas.

En este sentido, al superponer nuestros resultados del test de ansiedad en forma de decatipos, sobre los correspondientes una curva gaussiana bajo supuestos de normalidad, encontramos que, en hemodiálisis, son más abundantes las personas no sólo con valores máximos, sino también mínimos de ansiedad (ver Figura 3).

Pensamos que la presencia de alexitimia, alteración psicológica que se define como "alteración del estado de ánimo que cursa con dificultad para mostrar y comunicar los propios sentimientos", entre las personas en hemodiálisis podría explicar estos hallazgos. Este supuesto viene apoyado por otros autores que han podido asociar esta alteración anímica con el carácter crónico de la insuficiencia renal severa ${ }^{21-22 . ~ N o s ~ p r o p o n e m o s ~ l a ~}$ realización de futuros estudios para arrojar más luz sobre esta hipótesis en hemodiálisis. Consideramos que tener en cuenta este hecho es de gran importancia para la enfermería nefrológica, a la hora de detectar y poder derivar a una atención especializada a aquellas personas en hemodiálisis que necesiten de apoyo psicológico, ya que no siempre se van a encontrar entre las que se muestran más angustiadas o tristes, sino también entre aquellas en apariencia más indiferentes o impasibles. En estos casos, la asistencia psicológica les ayudaría a 
tomar conciencia y poder expresar sentimientos que, estando enmascarados, contribuirían, a la larga, a un mayor deterioro físico y mental en estas personas ${ }^{23}$.

A partir de estos resultados, consideramos que las necesidades de atención psicológica de las personas en hemodiálisis pueden ser evaluadas con diferentes herramientas como son los cuestionarios validados de depresión y ansiedad, u otros que nos aporten información sobre situaciones vitales distintas a la enfermedad, que constituyan potenciales agentes de sufrimiento en sus vidas $^{24}$. Dados los índices de ansiedad y depresión encontrados, así como la alta prevalencia de eventos potencialmente estresantes en sus vidas, concluimos sobre la necesidad de que las personas en hemodiálisis cuenten con un tratamiento donde la atención psicológica esté integrada.

Recibido: 30-10-18

Revisado: 15-12-18

Modificado: 17-02-19

Aceptado: 25-02-19

\section{Bibliografía}

1. Pérez J, Llamas F, Legido A. Insuficiencia renal crónica: revisión y tratamiento conservador. Archivos de Medicina 2005;1(3):1-10.

2. Arenas MD, Álvarez-Ude F, Angoso M, Berdud I, Antolín A, Lacueva J et al. Valoración del grado de dependencia funcional de los pacientes en hemodiálisis (HD): estudio multicéntrico. Nefrología 2006; 26(5):600-8.

3. Beck AT, Rush AJ, Shaw BF, Emery G. Cognitive Therapy of depression. New York: Guilford; 1979.

4. Bowlby J. La pérdida afectiva: tristeza y depresión. España: Paidós; 1993.

5. Valdés C, Ortega F. Avances en la calidad de vida relacionada con la salud y trasplante renal. Nefrología 2006;26(2):157-62.

6. Delacroix JM. Encuentro con la psicoterapia. Una visión antropológica de la relación y el sentido de la enfermedad en la paradoja de la vida. Francia: Cuatro Vientos; 2006.

7. Spielberg RD, Gorsuch RL, Lushene RE. Cuestionario de Ansiedad Estado-Rasgo STAI. Adaptación española por Buela-Casal G, Guillén-Riquelme A, Seisdedos N. España: TEA Ediciones; 2011.

8. Álvarez F, Fernández M, Vázquez AM, Mon C, Sánchez R, Rebollo P. Síntomas físicos y trastornos emocionales en pacientes en programa de hemodiálisis periódicas. Nefrología 2001;21(2):191-9.

9. Beck AT, Steer RA, Brown GK. Inventario de depresión de Beck Fast-Screen para pacientes médicos. Adaptación española por Sanz J, Izquierdo A y García-Vera MP. España: Pearson; 2011.

10. García FW, Fajardo C, Guevara R, Gonzáles V, Hurtado A. Mala adherencia a la dieta en hemodiálisis: el papel de los síntomas ansiosos y depresivos. Nefrología 2002;22(3):2245-52.

11. Drayer RA, Piraino B, Reynolds CF, Houck PR, Mazumdar S, Bernardini $J$ et al. Characteristics of depression in hemodialysis patients: symptoms, quality of life and mortality risk. Gen Hosp Psychiatry 2006 Jul-Aug; 28(4):306-12.

12. Cukor D, Peterson RA, Cohen SD, Kimmel PL. Depression in end-stage renal disease hemodialysis patients. Nat Clin Pract Nephrol 2006 Dec;2(12): 678-87.

13. García-Llana H, Remor E, Del Peso G, Selgas R. El papel de la depresión, la ansiedad, el estrés y la adhesión al tratamiento en la calidad de vida relacionada con la salud en pacientes en diálisis: revisión sistemática de la literatura. Nefrología 2014; 34(5):637-57.

14. Gómez L, Pac N, Manresa M, Lozano S, Chavarría JL. Prevalencia de ansiedad y depresión en pacientes de hemodiálisis. Enferm Nefrol 2015 AbrJun;18(2):112-7.

15. Páez $A E$, Jofre MJ, Azpiroz CR, De Bortoli MA. Ansiedad y depresión en pacientes con insuficiencia renal crónica en tratamientos de diálisis. Univ Psicol 2009;8:117-24. 
16. Muñoz R, Oto A, Barrio R, Fernández M. Evolución de la calidad de vida en pacientes en hemodiálisis: Estudio prospectivo a un año. Rev Soc Esp Enferm Nefrol 2006 Mar;9(1):55-8.

17. Moreno E, Arenas MD, Porta E, Escalant L, Cantó MJ, Castell $G$ et al. Estudio de la prevalencia de trastornos ansiosos y depresivos en pacientes en hemodiálisis. Rev Soc Esp Enferm Nefrol 2004;7(4):225-33.

18. Contreras F, Espinosa JC, Esguerra GA. Calidad de vida, autoeficacia, estrategias de afrontamiento y adhesión al tratamiento en pacientes con insuficiencia renal crónica sometidos a hemodiálisis. Psicología y Salud 2008;18:165-79.

19. Calvanese N, Feldman L, Weisinger J. Estilos de afrontamiento y adaptación al tratamiento en pacientes sometidos a hemodiálisis. Nefrol Latín 2004;11:49-63.
20. Schnake A. Los diálogos del cuerpo. El enfoque holístico de la enfermedad. Chile: Cuatro Vientos; 1995.

21. Kojima M, Hayano J, Suzuki S, Seno H, Kasuga $\mathrm{H}$, Takahashi $\mathrm{H}$ et al. Depression, Alexithymia and Long-Term Mortality in Chronic Hemodialysis Patients. Psychotherapy and Psychosomatics 2010;79 (5):303-11.

22. Lumley MA, Stettner L, Wehmer F. How are alexithymia and physical illness linked? A review and critique of pathways. J Psychosom Res 1996;41(6):505-18.

23. Schnake A. Enfermedad, síntoma y carácter. Diálogos gestálticos con el cuerpo. Chile: Cuatro Vientos; 2007.

24. Motrico E, Rodero ML, Álvarez J, Salinas JA, Moreno $P$. Measures for de assessment of stressful life events in the Spanish adult population: A systematic review. Anales de Psicología 2017;33(3):556-67. 


\section{Anexo 1. Cuestionario de experiencias vitales}

\section{$\overline{B L O Q U E ~ I N F A N C I A ~}$}

¿Qué información tiene acerca de su embarazo y nacimiento?

¿Qué lugar ocupa dentro de su sistema familiar?

¿Qué aspectos destacaría de su infancia, considerando este período de los 0 a 12 años?

¿Hubo alguna situación de conflicto o estresante para usted que quiera resaltar?

DCómo definiría la relación con sus padres y hermanos (en caso de tenerlos) durante este período?

¿Durante cuánto tiempo fue a la escuela?, en caso de no ir, ¿a qué se debió?

¿Qué recuerda de su relación con otros niños de su edad durante este período?

¿Padeció alguna enfermedad importante durante este período?

\section{BLOQUE JUVENTUD}

¿Qué aspectos destacaría de su juventud, teniendo en cuenta este período de los 12 a los 20 años?

¿ ¿Tuvo lugar alguna situación de conflicto o estresante para usted que quiera resaltar durante este período?

¿Qué acontecimientos destacaría en relación a su vida sentimental en este período?

¿Tuvo acceso a la escuela y/o se formó durante este período?

¿Padeció alguna enfermedad importante durante este período?

\section{BLOQUE ACTUALIDAD}

¿Qué acontecimientos vitales destacaría de los que hayan tenido lugar a partir de los 20 años?

D En caso de que sus padres hayan fallecido ¿qué edad tenían al fallecer y cuál fue el motivo?

D Si aún está vivo alguno de ellos o los dos ¿dónde y con quién vive/n, cómo se encuentra/n y cuál es su nivel de autonomía?

D Actualmente, ¿cuál es su situación familiar?

¿Qué acontecimientos destacaría en relación a su vida sentimental actual?

¿Tiene hijos?, en caso afirmativo ¿Qué aspectos destacaría en cuanto a la relación con ellos? ¿Ha ocurrido algún acontecimiento a resaltar en la vida de sus hijos que le haya afectado de forma significativa (positiva o negativamente)?

¿Ha trabajado o trabaja actualmente? En caso afirmativo, ¿Qué acontecimientos destacaría sobre su vida laboral?

¿Con qué ingresos o recursos económicos cuenta actualmente?

¿Tiene amigos en la actualidad? ¿Qué actividades sociales y/o de ocio realiza actualmente?

¿Qué enfermedad/es ha padecido a partir de los 20 años?, ¿Cuál/es padece actualmente?

¿Alguno de sus familiares cercanos padece alguna enfermedad importante y/o crónica?

\section{Estrategias de afrontamiento}

Cuando surge algún problema o conflicto en su vida, a cuál de estas estrategias de afrontamiento diría que recurre con más frecuencia (elija una o dos a lo sumo de entre las opciones que se ofrecen):

a) Trato de encontrar alguien que me apoye o con quien desahogarme.

b) Trato de no pensar en ello, busco formas de distraerme y mantenerme ocupado.

c) Me siento muy enfadado e irritable, y los demás me lo notan.

d) No puedo evitar sentirme culpable y/o involucrado en lo que ocurre y trato de buscar soluciones a toda costa.

e) Me focalizo en la parte positiva de lo ocurrido para encontrar una salida al problema y aprender de ello.

f) Me siento muy impotente, me bloqueo y me resulta difícil encontrar algo que hacer al respecto.

Este artículo se distribuye bajo una Licencia Creative Commons Atribución-NoComercial 4.0 Internacional. https://creativecommons.org/licenses/by-nc/4.0/

Open Access (c) (7) (8) 